\section{Contus} Thomas' Hospital, Guy's and St Thomas' NHS Foundation Trust, London, UK

${ }^{2}$ Department of Respiratory, Whittington Hospital, London, UK

${ }^{3}$ Guy's and St Thomas' NHS Foundation Trust, London, UK

\section{Correspondence to} Dr Hugh Ip, Respiratory Registrar, Lane Fox Respiratory Service, St Thomas' Hospital, Guy's and St Thomas' NHS Foundation Trust, Westminster Bridge Road, London SE1 7EH, UK; Hughip@gmail.com

HI and WLW are joint first authors.

Received 21 November 2016 Revised 6 January 2017

Accepted 26 January 2017 Published Online First 23 February 2017
CrossMark

To cite: Ip $\mathrm{H}$, Woo WL,

Darakhshan A, et al. Thorax

2017;72:1169-1170.

\title{
Let air out of the bowel to allow more air in the lungs: surgical treatment of weaning failure
}

\author{
Hugh Ip, ${ }^{1}$ Wen Ling Woo, ${ }^{2}$ Amir Darakhshan, ${ }^{3}$ Nicholas Hart ${ }^{1}$
}

A 79-year-old man was electively admitted for a Whipple's procedure for a pancreatic cancer. Following surgery, he was extubated, but subsequently developed a hospital-acquired pneumonia with multiple organ failure requiring admission to the intensive care unit for organ support. After a protracted period of failed weaning from mechanical ventilation, he was transferred to a regional weaning centre 7 months after the initial operation. Since the surgery, the patient had complained of abdominal bloating and discomfort with distended bowel loops observed on the plain abdominal film. Because of frailty of the patient, a conservative management approach was taken, including high positive extrinsic pressure to enhance lung volume and the use of prokinetics, low-volume enteral feeding, regular aspiration of air from the nasogastric tube, sitting out and mobilisation, a bowel management regime, pancreatic enzyme replacement and high-dose cholinesterase inhibitor to promote peristalsis and reduce bowel distension. The patient complained of dyspnoea associated with abdominal distension and the chest radiograph demonstrated reduced lung volumes and bilateral hemidiaphragm elevation with a grossly distended large bowel (figure 1A). The symptoms persisted despite surgical management, and a surgical review was sought with cross-sectional imaging performed. A CT of the chest, abdomen and pelvis showed dilated large bowel loops throughout the whole length of the bowel measuring up to $8.6 \mathrm{~cm}$ with no transition point (figure 2A). A flexible sigmoidoscopy failed to significantly decompress the sigmoid and descending colon, confirming the diagnosis of chronic colonic pseudo-obstruction. Due to ongoing symptoms, the patient consented to percutaneous endoscopic colostomy (PEC) to mechanically decompress the colon of gas and retained faeces. Two $20 \mathrm{~F}$ PEC tubes were inserted into the sigmoid colon with the position checked by digital pressure, transillumination of the sigmoid colon and direct visualisation with endoscopy. A week after the procedure, a chest radiograph showed significant re-expansion of both lungs and resolution of distended bowel loops (figure 1B). A CT of the abdomen and pelvis demonstrated two PEC tubes sited within the colon with normal-calibre large bowel and a small volume of intraperitoneal free gas secondary to the procedure, as expected (figure 2B). The images were reflected in the patient's condition with marked reduction in abdominal bloating, reduced dyspnoea and reduced ventilator requirements. The patient is currently undergoing a combined weaning and rehabilitation programme

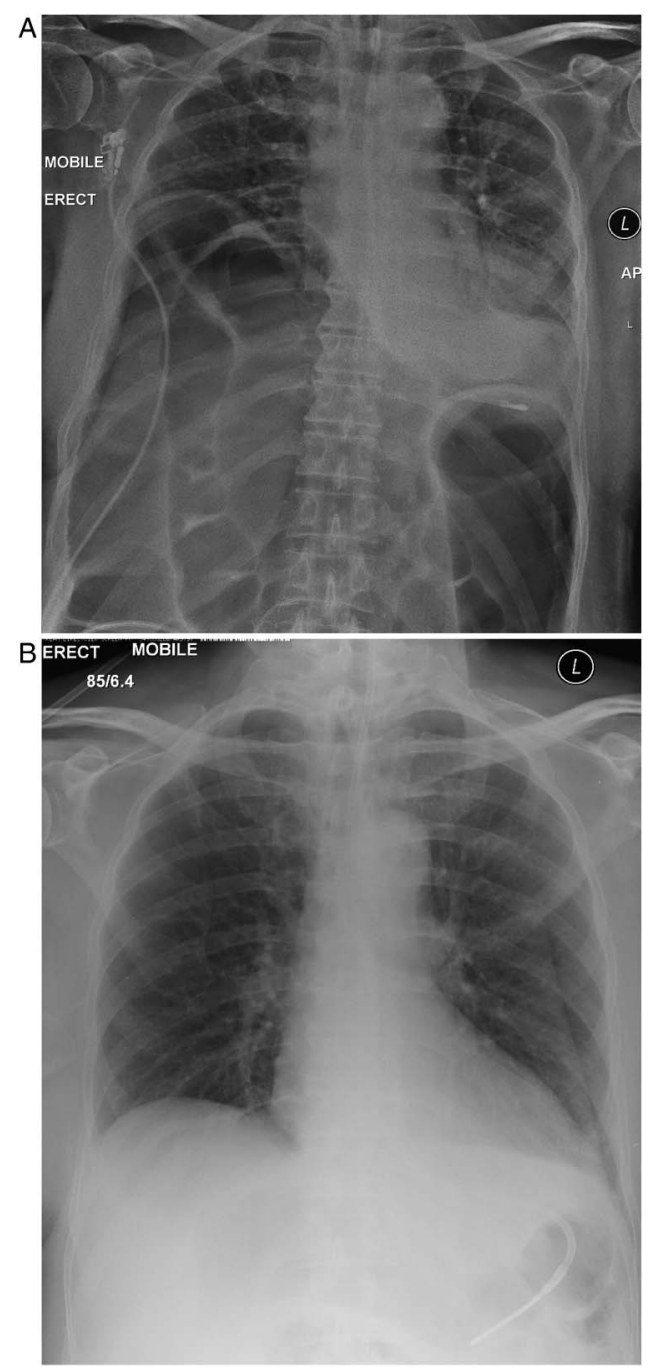

Figure 1 (A) Chest radiograph demonstrating small lung volumes and dilated loops of bowel. (B) Chest radiograph following percutaneous endoscopic colostomy tube insertion with resolution of dilated loops of bowel and increase in lung volumes.

with a gradual reduction in pressure support with cuff-down trials. He is expected to wean completely from invasive mechanical ventilation.

PEC was first described in $1986^{1}$ with guidance on its use issued by the National Institute for Health and Care Excellence in 2006. ${ }^{2}$ This case illustrates the potential novel use of PEC in patients with severe colonic distension who are refractory to conservative measures, leading to diaphragmatic splinting, and impaired weaning from mechanical ventilation. Indeed, reducing the respiratory muscle elastic load 


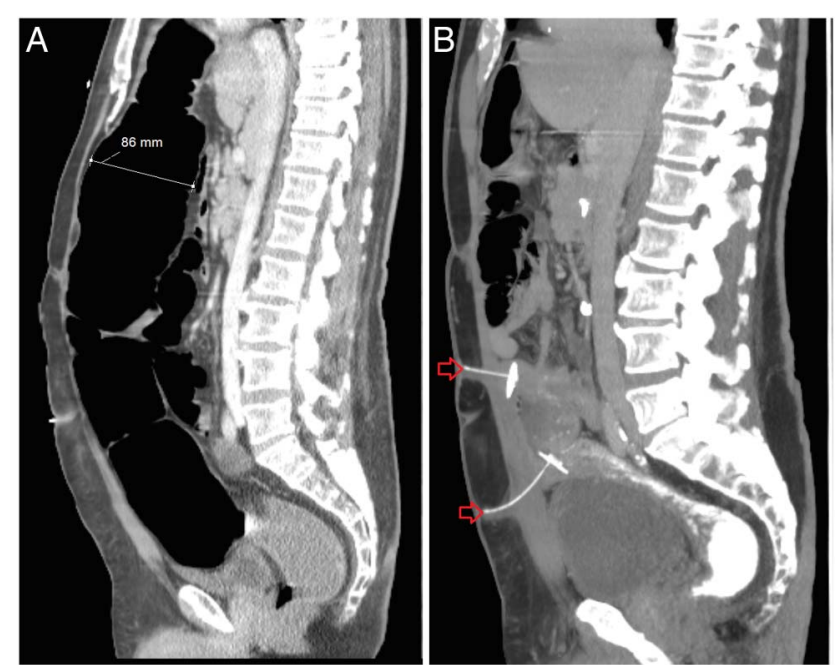

Figure 2 (A) Sagittal CT section demonstrating dilatation of large bowel loops $(8.6 \mathrm{~cm})$. (B) Sagittal CT section demonstrating two percutaneous endoscopic colostomy tubes in situ (red arrows) with resolution of dilated loops of bowel. caused by the abdominal distension was highly effective in progressing weaning in this patient, ${ }^{3}$ with presumed improvement in diaphragm function as has been previously reported with colectomy. ${ }^{4}$

Twitter Follow Nicholas Hart @NickHartThorax

Contributors $\mathrm{NH}, \mathrm{AH}$ and $\mathrm{HI}$ managed the patient. $\mathrm{HI}$ and WLW drafted the manuscript. WLW prepared the images. All authors reviewed and revised the manuscript.

Competing interests None declared.

Patient consent Obtained.

Provenance and peer review Not commissioned; externally peer reviewed.

\section{REFERENCES}

1 Ponsky JL, Aszodi A, Perse D. Percutaneous endoscopic cecostomy: a new approach to nonobstructive colonic dilation. Gastrointest Endosc 1986;32:108-11.

2 Percutaneous endoscopic colostomy (Interventional procedure guidance). National Institute for Health and Care Excellence, 2006. http://www.nice.org.uk/guidance/ ipg161 (accessed 31 Oct 2016).

3 Hart N. Respiratory failure. Medicine 2008;36:242-5.

4 Nava S, Schiavoni PG. Hemidiaphragm dysfunction due to megacolon: functional recovery after colectomy. Monaldi Arch Chest Dis 1995;50: 18-20. 